\title{
Haploinsufficiency of the Nijmegen breakage syndrome 1 gene increases mammary tumor latency and metastasis
}

\author{
ROWENA WAN and DAVID L. CROWE \\ University of Illinois Cancer Center, Chicago, IL 60612, USA
}

Received March 8, 2012; Accepted April 9, 2012

DOI: $10.3892 /$ ijo.2012.1435

\begin{abstract}
Human diseases such as Nijmegen breakage syndrome due to mutations in the NBS1 gene result in defects in resection of double strand breaks. NBS1 functions as part of the MRN complex which functions in homologous recombination and non-homologous end joining. NBS is a rare human autosomal recessive disorder caused by hypomorphic mutations. At the cellular level, NBS is characterized by radiosensitivity, chromosomal breakage and defective cell cycle checkpoints. NBS1 null mutations result in early embryonic lethality in mice, but NBS1 hypomorphic mutants are viable. Cells from these mice are defective in $\mathrm{S}$ phase and $\mathrm{G} 2 / \mathrm{M}$ checkpoints. In humans, NBS1 polymorphisms have been associated with increased risk of breast cancer. MRN expression was reduced in the majority of breast tumors, and low expression of MRN correlated with increased histologic grade and estrogen receptor negativity. While these studies have shown NBS1 to be important in clinical outcomes of patients with breast cancer, mammary tumors are rare in the NBS1 haploinsufficient mouse. To better understand the role of NBS1 in mammary tumorigenesis, we examined the NBS1+/-;MMTV-neu mouse model. Mammary tumor latency was markedly increased in NBS1+/-;neu mice compared to NBS1+/+;neu control animals. This effect was due to increased apoptosis in early NBS1+/-;neu mammary tumors. However, NBS1+/-;neu mammary tumors were highly metastatic and demonstrated clear differences in gene expression profiles compared to control tumors. We concluded that NBS1 haploinsufficiency results in increased mammary tumor latency and metastasis.
\end{abstract}

\section{Introduction}

Double strand break repair is mediated by two major repair pathways, homologous recombination (HR) or non-homologous end joining (NHEJ) (1). In mammalian cells more than $90 \%$

Correspondence to: Dr David L. Crowe, University of Illinois Cancer Center, 801 S. Paulina St., Room 530C, Chicago, IL 60612, USA

E-mail: dlcrowe@uic.edu

Key words: Rad50, Mre11, Nijmegen breakage syndrome 1 gene, MMTV-neu, DNA damage of double strand breaks are repaired by NHEJ. Both pathways are defined and their impairment is associated with cell cycle arrest, cell death, genomic instability, and cancer (2). Human diseases such as Nijmegen breakage syndrome due to mutations in the NBS1 gene result in defects in resection of double strand breaks (3). NBS1 functions as part of the MRN complex whose functions are not restricted to HR but are also involved NHEJ (4).

NBS is a rare human autosomal recessive disorder caused by hypomorphic mutations. This disorder is characterized by growth retardation, immunodeficiency, microcephaly, and cancer predisposition. At the cellular level, NBS is characterized by radiosensitivity, chromosomal breakage, and defective cell cycle checkpoints. NBS1 null mutations in mice result in early embryonic lethality (5), but NBS1 hypomorphic mutants are viable (6). Cells from these mice are defective in $S$ phase and G2/M checkpoints. Heterozygous mice with an NBS1 null mutation and homozygous animals with hypomorphic mutations are predisposed to different types of cancer. Conditional NBS1 mutant mice also have been characterized (7). For example, neuronal inactivation of NBS1 results in chromosomal breaks, microcephaly, growth retardation, cerebellar defects, and ataxia.

NBS1 polymorphisms have been associated with increased risk of breast cancer (8-10). MRN expression was reduced in the majority of breast tumors (11), and low expression of MRN correlated with increased histologic grade and estrogen receptor negativity. Response to radiotherapy correlated with high expression of the MRN complex. Patients with high numbers of ionizing radiation induced NBS1 foci had aggressive breast cancer phenotypes $(12,13)$. While these studies have shown NBS1 to be important in clinical outcomes of patients with breast cancer, mammary tumors are rare in the NBS1 haploinsufficient mouse. To better understand the role of NBS1 in mammary tumorigenesis, we characterized these cancers using the NBS1+/-;MMTV-neu mouse.

\section{Materials and methods}

Transgenic mouse procedures. Animal procedures were approved by the Institutional Animal Care Committee. NBS1+/- mice (6) were crossed with the mammary tumor prone MMTV-neu transgenic strain in the FVB background (The Jackson Laboratory, Bar Harbor, ME). Tumor development in $30 \mathrm{NBS1+/-;neu} \mathrm{mice} \mathrm{was} \mathrm{compared} \mathrm{to} \mathrm{that} \mathrm{in} 30 \mathrm{NBS1+/+;neu}$ 
control mice. Mice were genotyped by PCR analysis of tail DNA samples. The mammary gland chains of female mice were examined visually and by palpation twice weekly. Tumors were measured twice weekly using calipers. Mice were euthanized 4 or 8 weeks after tumor formation followed by complete necropsy. Tumor tissue was processed for histopathologic and gene expression analyses. Statistical analysis was determined by ANOVA.

Mammary gland and tumor histopathology. For whole mount analysis, mammary glands were fixed in 3:1 ethanol:acetic acid, mounted on microscope slides, and stained with carmine-alum followed by clearing in 2:1 benzyl benzoate:benzyl alcohol. Tumor tissue was fixed in formalin for $16 \mathrm{~h}$ at room temperature. Tissue was dehydrated in an ethanol series followed by clearing in xylene and embedding in paraffin. Five micrometer sections were cut from the blocks and placed on poly-L-lysine coated slides. Sections were deparaffinized in xylene and stained with hematoxylin and eosin for histopathologic interpretation.

Cellular proliferation and programmed cell death analysis. Mouse mammary tumor tissue was dissociated to single cells by trypsinization, fixed with $2 \%$ formaldehyde for $20 \mathrm{~min}$ at $4^{\circ} \mathrm{C}$ followed by permeabilization with $70 \%$ ethanol, then washed with PBS. For proliferation analysis, cells were incubated with anti-PCNA antibody followed by anti-rabbit IgG secondary antibody conjugated to fluorescein. Cells were washed extensively with PBS. For programmed cell death analysis, cells were incubated with terminal deoxynucleotidyl transferase and fluorescein conjugated dUTP at $37^{\circ} \mathrm{C}$ for $30 \mathrm{~min}$ followed by washing in PBS. The percentage of fluorescein positive cells in each group was determined by flow cytometry.

Reverse transcription-polymerase chain reaction. RNA was extracted from mouse mammary tumors using a commercially available kit (Qiagen, Valencia, CA) and reverse transcribed using SuperScript II reverse transcriptase according to manufacturer's instructions (Invitrogen, Carlsbad, CA). cDNA was amplified using specific primers (5'-3'): Nbs1, TTCCCCCAT ACAAGTATCCAG and AACTTAAGGAGCATCTATGCAG; Malat1, CATCCCGTTCCTTGTTACTC and AGACTACAA ACATTGTGTCGTG; Cdkn2b, GCTAAATGGGAAACC TGGAGAG and ACGTTGAGTCTGTCAGAATCC; Hifla, CAGCTCCCTTTCTGATAAGC and TCTTCAGTTTCTGT GTCATCG; Egf, TCTGTCAACCCCTGAATAAATG and TTTCACTGGGAAAGACTTCAAG; Lalba, TACCCTGTA GTGACACCACC and TAAAACCCCCATCGAGACC; Jag1, AATGCTGAACCACTTGTAGAC and GGTGAACCT GGATCACTCTG; Areg, TGAATCATTGCCAAGCCAC and TAAAAAGTGACAACTGGGCATC; Csn1s1, CTCCATCC ACCTCATGTCTC and CGCTCAGATGATGCAACTG; Tgfb2, ATGTCTTCAGCCGAGGTCTG and CCCACATC TTCTTTCTCTGCTC; Ccnb2, GTATTACACAGGCTAC ATGGAG and ACATACAGGATCTGAGAAGCG; Dsc2, ATGCTGTGCCTTGCTTTAG and AGCATTTGGTGT TCACAGAC; Csn1s2, TCTTCGTGGTTTCCCCATC and ACTTTAATGTCTTGGCGAGAG in $20 \mathrm{mM}$ Tris- $\mathrm{HCl}$ (pH 8.3), $1.5 \mathrm{mM} \mathrm{MgCl}_{2}, 63 \mathrm{mM} \mathrm{KCl}, 0.05 \%$ Tween $20,1 \mathrm{mM}$ EGTA, $50 \mu \mathrm{M}$ of each dNTP, and 2.5 U Taq DNA polymerase (Roche Applied Science, Indianapolis, IN). Amplification with $\beta$-actin cDNA using primers 5'-ACAGGAAGTCCCTTGCC ATC-3' and 5'-ACTGGTCTCAAGTCAGTGTACAGG-3' as the internal control was carried out by real-time PCR (iCycler, Bio-Rad) using cycle parameters $94^{\circ} \mathrm{C}$ for $25 \mathrm{sec}, 55^{\circ} \mathrm{C}$ for $1 \mathrm{~min}$, and $72^{\circ} \mathrm{C}$ for $1 \mathrm{~min}$.

Global gene expression profiling. Total RNA was extracted from microdissected tumors ( $\mathrm{n}=3$ for each group) using a commercially available kit (RNEasy, Qiagen). Integrity of ribosomal RNA bands was confirmed by northern gel electrophoresis. For each hybridization, total RNA (10 $\mu \mathrm{g})$ was converted to labeled cRNA targets. The biotinylated cRNA targets were then purified, fragmented, and hybridized to GeneChip mouse genome 4302.0 expression arrays (Affymetrix, Santa Clara, CA) to interrogate the abundance of 39,000 possible transcripts in each sample. Affymetrix GCOS software was used to generate raw gene expression scores and normalized to the relative hybridization signal from each experiment. All gene expression scores were set to a minimum value of 2 times the background determined by GCOS software in order to minimize noise associated with less robust measurements of rare transcripts. Data was analyzed by $\mathrm{t}$-test with $\mathrm{p}<0.005$ followed by ratio analysis (minimum 2-fold change).

\section{Results}

The histopathology of NBS1+/-;neu mammary glands was consistent with that of NBS1+/+;neu animals, exhibiting normal mammary ductal structure in young mice (Fig. 1A). Mice from both genotypes developed hyperplastic terminal end buds which progressed to poorly differentiated adenocarcinoma (Fig. 1B). Monomorphic sheets of poorly differentiated epithelial cells with duct formation was observed in tumors from both NBS1+/-;neu and NBS1+/+;neu mice (Fig. 1C and D). Lung metastasis was observed in both groups of animals (Fig. 1E). The tumor latency in NBS1+/-;neu mice was dramatically increased (77 weeks vs. 40 weeks for NBS1+/+;neu animals; p $<0.01$; Fig. 2A). However NBS1+/-;neu mammary tumors were highly metastatic (4-fold increase in percentage of mice with metastatic tumors $(\mathrm{p}<0.02$; Fig. 2B).

To understand the increased latency of NBS1+/-;neu mammary tumors, we analyzed cellular proliferation and programmed cell death. As shown in Fig. 3A, 4 week NBS1+/-;neu tumors exhibited a 7-fold increase in apoptotic cells as determined by TUNEL analysis. The percentage of apoptotic cells in eight week NBS1+/-;neu tumors was similar to that in NBS1+/+;neu mice. We also analyzed cellular proliferation in early and late stage NBS1+/-;neu and NBS1+/+;neu mammary tumors. We did not detect significant differences in the percentages of PCNA positive cells between these groups (Fig. 3B). We concluded that increased apoptosis in NBS1+/-;neu mammary tumors resulted in increased latency.

To understand the differences in mammary tumor phenotype in NBS1+/-;neu mice, we performed global gene expression profiling. Changes in cancer gene expression are shown in Tables I-III. Primary tumors from NBS1+/-;neu and NBS1+/+;neu were more highly related to each other than to metastatic tumors. Bioinformatic analysis revealed gene expression changes in specific pathways distinguishing NBS1+/-;neu from NBS1+/+;neu primary tumors, including metastasis 

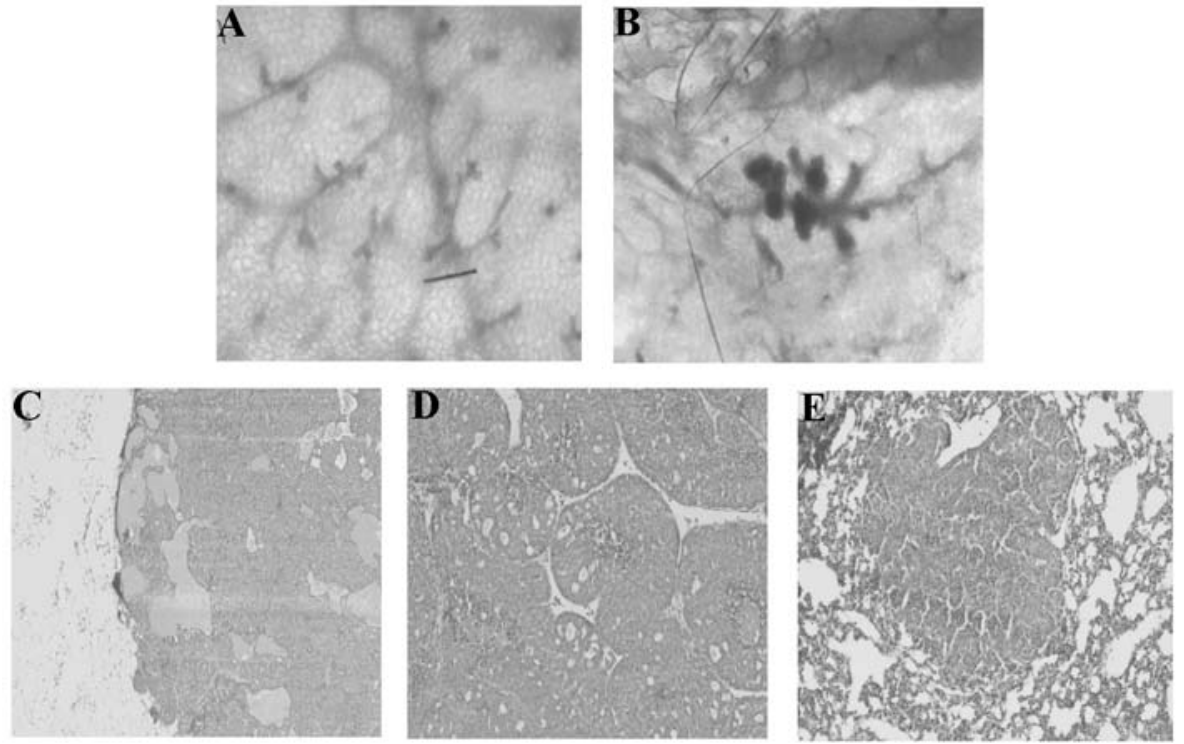

Figure 1. NBS1 haploinsufficiency increases tumor latency in the mammary tumor prone MMTV-neu mouse. (A) Whole mount preparation of mammary fat pad in NBS1+/- mouse. (B) Whole mount preparation of mammary fat pad in NBS1+/-;neu mouse showing hyperplastic terminal end buds. (C) Poorly differentiated adenocarcinoma in the mammary fat pad of NBS1+/-;neu mouse. Section is stained with hematoxylin and eosin; scale bar $=100 \mu \mathrm{m}$. (D) Higher magnification of poorly differentiated adenocarcinoma in NBS1+/-;neu mouse. Tumor consists of sheets of poorly differentiated epithelial cells and ducts. Scale bar $=50 \mu \mathrm{m}$. (E) Metastatic tumor in lung of NBS1+/-;neu mouse. Magnification is same as in (D).

A
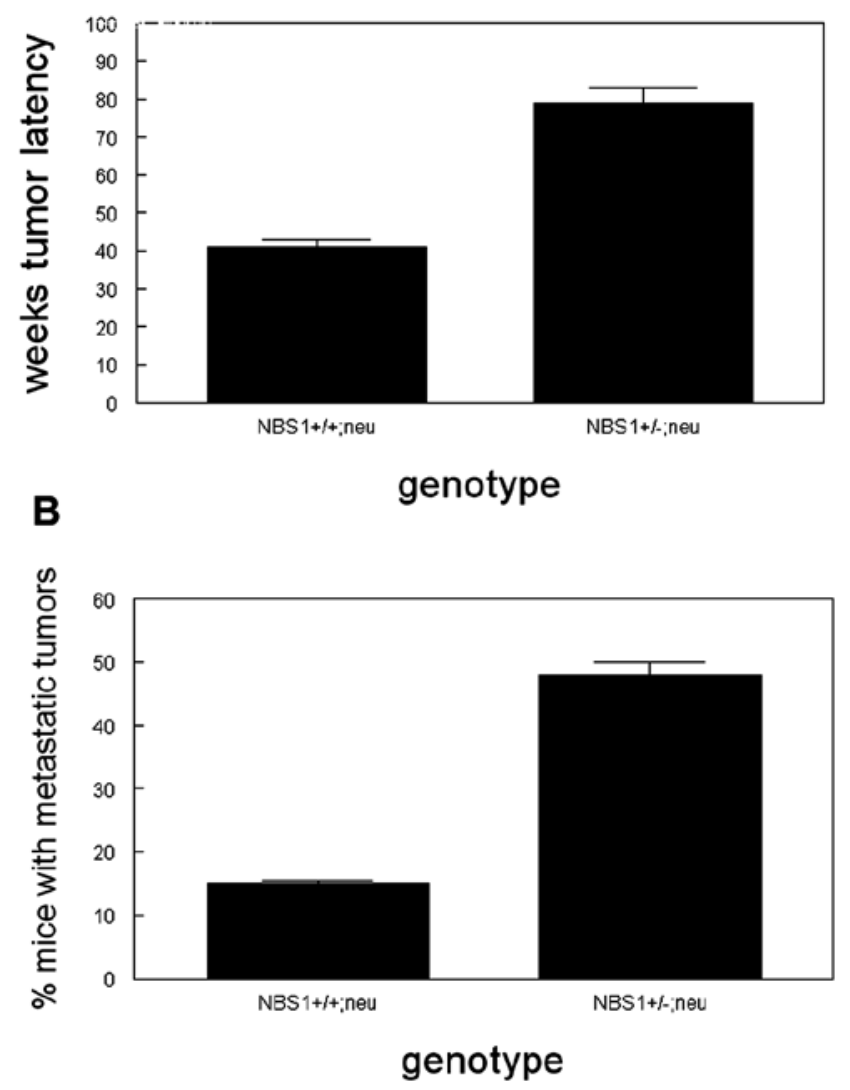

Figure 2. (A) Increased tumor latency in NBS1+/-;neu mice. The number of weeks to tumor formation in NBS1+/+;neu and NBS+/-;neu mice were determined by twice weekly palpation. (B) Increased metastatic mammary cancer in NBS1+/-;neu mice. The percentage of NBS1+/-;neu and NBS1+/+;neu mice with metastatic mammary tumors is shown. Error bars indicate SEM.
A

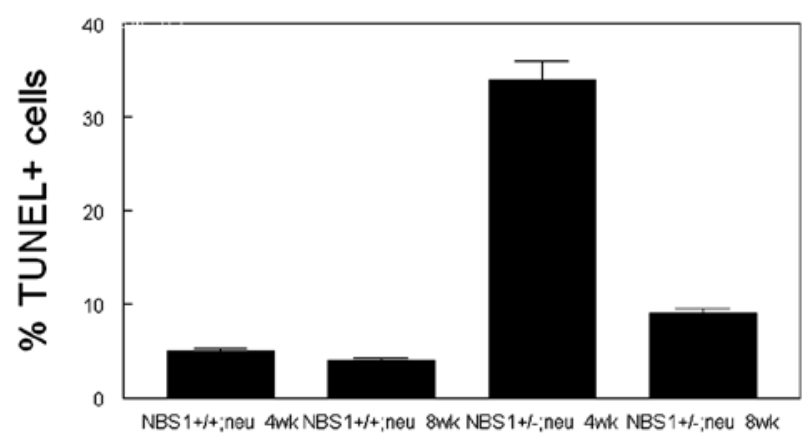

B

\section{tumors}

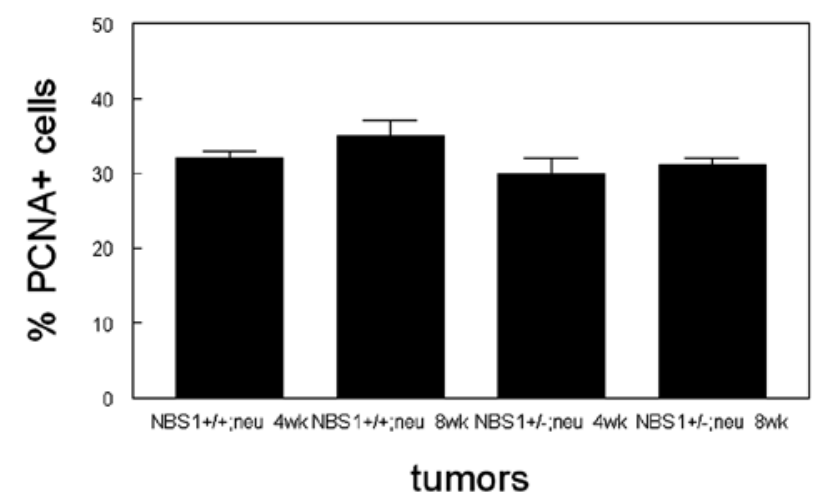

Figure 3. Increased programmed cell death in early NBS1+/-;neu mammary tumors correlates with increased latency. (A) The percentage of apoptotic cells in early and late stage NBS1+/-;neu and NBS1+/+;neu mammary tumors was determined as described in Materials and methods. (B) To assess cellular proliferation, the percentage of PCNA positive cells in early and late stage NBS1+/-;neu and NBS1+/+;neu mammary tumors was determined. Error bars indicate SEM. 
Table I. Differentially expressed genes between NBS1+/+;neu and NBS+/-;neu primary mammary tumors (55 genes).

\begin{tabular}{|c|c|c|c|}
\hline Accession & Gene name & Gene symbol & Fold change \\
\hline NM_007470 & Apolipoprotein D & Apod & 796.0 \\
\hline AV325919 & ATPase, $\mathrm{Na}^{+} / \mathrm{K}^{+}$transporting, $\alpha 2$ & Atp1a2 & 198.9 \\
\hline AF108501 & Chloride channel calcium activated 2 & Clca2 & 58.0 \\
\hline NM_030677 & Glutathione peroxidase 2 & Gpx2 & 54.1 \\
\hline AF146523 & Metastasis lung adenocarcinoma 1 & Malat1 & 43.6 \\
\hline NM_008183 & Glutathione S-transferase, mu 2 & Gstm1 & 31.8 \\
\hline NM_134032 & Homeo box B2 & Hoxb2 & 18.0 \\
\hline ВС011063 & Homeo box A5 & Hoxa5 & 17.1 \\
\hline AW105779 & Lactate dehydrogenase D & Ldhd & 14.6 \\
\hline AV367068 & Desert hedgehog & Dhh & 13.8 \\
\hline NM_013605 & Mucin 1, transmembrane & Muc1 & 12.2 \\
\hline AF059567 & Cyclin-dependent kinase inhibitor 2B & Cdkn2b & 11.4 \\
\hline NM_008259 & Forkhead box A1 & Foxa1 & 10.5 \\
\hline NM_008608 & Matrix metallopeptidase 14 & Mmp14 & 10.3 \\
\hline AI649186 & Fibroblast growth factor 1 & Fgf1 & 9.6 \\
\hline U30244 & Ephrin B2 & Efnb2 & 9.5 \\
\hline BE686893 & Nuclear receptor coactivator 7 & Ncoa7 & 9.0 \\
\hline AB049755 & Mannan-binding lectin serine peptidase & Masp1 & 9.0 \\
\hline BG073383 & Homeo box B3 & Hoxb3 & 8.9 \\
\hline NM_008858 & Protein kinase $\mathrm{C}, \mathrm{mu}$ & Prkcm & 8.5 \\
\hline BB197591 & Protocadherin 7 & $\operatorname{Pcdh} 7$ & 8.4 \\
\hline BB224034 & Phospholipase C, $\beta 4$ & Plcb4 & 8.1 \\
\hline BB322941 & Nuclear receptor subfamily 4 , group A, 2 & $\mathrm{Nr} 4 \mathrm{a} 2$ & 7.4 \\
\hline NM_007556 & Bone morphogenetic protein 6 & Bmp6 & 7.0 \\
\hline NM_013867 & Breast cancer anti-estrogen resistance 3 & Bcar3 & 6.8 \\
\hline BB269715 & Hypoxia inducible factor $1, \alpha$ subunit & Hif1a & 6.4 \\
\hline BB795235 & Frizzled homolog 5 (Drosophila) & Fzd5 & 6.4 \\
\hline BB795491 & DNA methyltransferase 3A & Dnmt3a & 6.3 \\
\hline NM_007631 & Cyclin D1 & Ccnd1 & 6.3 \\
\hline ВC024375 & Growth hormone receptor & Ghr & 6.2 \\
\hline AA214868 & Phosphatase and tensin homolog & Pten & 6.2 \\
\hline BB520860 & RAR-related orphan receptor $\alpha$ & Rora & 6.2 \\
\hline BB376407 & Jumonji, AT rich interactive domain $1 \mathrm{~A}$ & Jarid1a & 5.9 \\
\hline AV359819 & Jagged 1 & Jag1 & 5.5 \\
\hline NM_008054 & Fyn proto-oncogene & Fyn & 5.4 \\
\hline AV241297 & Serine peptidase inhibitor, Kazal type 5 & Spink5 & -6.4 \\
\hline NM_010113 & Epidermal growth factor & Egf & -8.3 \\
\hline NM_010679 & Lactalbumin, $\alpha$ & Lalba & -21.7 \\
\hline NM_009973 & Casein $\alpha$ s2-like B & Csn $1 \mathrm{~s} 2 \mathrm{~b}$ & -27.2 \\
\hline NM_008644 & Mucin 10 , salivary mucin & Muc10 & -100.4 \\
\hline
\end{tabular}

(Malat1, 43.6-fold; Mmp14, 10.3-fold), cell cycle (Cdkn2b, 11.4fold), angiogenesis (Hifla, 6.4-fold), epidermal growth factor signaling (Egf, -8.3-fold), and differentiation (Lalba, -21.7-fold; Csn1s2b,-27.2-fold; Muc10,-100.4-fold). We also compared gene expression in NBS1+/-;neu and NBS1+/+;neu metastatic tumors. These pathways included metastasis, Notch signaling (Dlk1, 462.9-fold; Jag1, 28.6-fold; Jag2, 6.2-fold), epidermal growth factor receptor signaling (Areg, 66.3-fold; Ereg, 23.5-fold; Hbegf, 9.4-fold), fibroblast growth factor signaling (Fgfr2, 68.9- fold; Fgf1, 14.9-fold; Fgfr3, 10.9-fold), peroxisome proliferator activated receptor signaling (Pparg, 21.8-fold; Ppara, 10.1-fold, Ppargclb, 7.1-fold), forkhead signaling (Foxa2, 42.6-fold; Foxa1, 33.6-fold; Foxp2, 19-fold; Foxq1, 18.1-fold), and differentiation (Wap, -22.1-fold; Csn3, -32.3-fold; Lalba, -212.2-fold; Csn, -310.8-fold; Csn1s1, -365.2-fold). Finally we compared gene expression in primary and metastatic NBS1+/-;neu mammary tumors. These pathways included Notch signaling (Dlk1, 308.8fold; Jag1, 13.9-fold; Jag2, 6.2-fold), transforming growth factor/ 
Table II. Differentially expressed genes between NBS1+/+;neu and NBS1+/-;neu metastatic mammary tumors (239 genes).

\begin{tabular}{|c|c|c|c|}
\hline Accession & Gene name & Gene symbol & Fold change \\
\hline NM_010052 & $\Delta$-like 1 homolog (Drosophila) & Dlk1 & 462.9 \\
\hline NM_007663 & Cadherin 16 & Cdh16 & 140.1 \\
\hline NM_008471 & Keratin 19 & Krt19 & 98.6 \\
\hline AF146523 & Metastasis lung adenocarcinoma 1 & Malat1 & 69.8 \\
\hline NM_010207 & Fibroblast growth factor receptor 2 & Fgfr2 & 68.9 \\
\hline NM_009704 & Amphiregulin & Areg & 66.3 \\
\hline AV304616 & Sonic hedgehog & Shh & 44.6 \\
\hline NM_010446 & Forkhead box A2 & Foxa2 & 42.6 \\
\hline NM_007554 & Bone morphogenetic protein 4 & Bmp4 & 34.7 \\
\hline BF585144 & Regulator of G-protein signaling 5 & Rgs5 & 34.1 \\
\hline NM_008259 & Forkhead box A1 & Foxa1 & 33.6 \\
\hline AV022238 & Chemokine (C-X-C motif) ligand 15 & Cxcl15 & 32.7 \\
\hline NM_008398 & Integrin $\alpha 7$ & Itga7 & 30.2 \\
\hline AV359819 & Jagged 1 & Jag1 & 28.6 \\
\hline NM_007950 & Epiregulin & Ereg & 23.5 \\
\hline NM_011146 & Peroxisome proliferator receptor $\gamma$ & Pparg & 21.8 \\
\hline U30244 & Ephrin B2 & Efnb2 & 21.4 \\
\hline NM_133721 & Integrin $\alpha 9$ & Itga9 & 20.7 \\
\hline ВC002073 & Chemokine (C-C motif) ligand 6 & $\mathrm{Ccl} 6$ & 19.6 \\
\hline NM_019932 & Chemokine (C-X-C motif) ligand 4 & Cxcl4 & 19.4 \\
\hline NM_017399 & Fatty acid binding protein 1 , liver & Fabp & 19.4 \\
\hline AV322952 & Forkhead box P2 & Foxp2 & 19.0 \\
\hline AV009267 & Forkhead box Q1 & Foxq1 & 18.1 \\
\hline AI649186 & Fibroblast growth factor 1 & Fgf1 & 14.9 \\
\hline NM_013867 & Breast cancer anti-estrogen resistance 3 & Bcar3 & 13.1 \\
\hline AV239587 & Bone morphogenetic protein 2 & Bmp2 & 11.8 \\
\hline NM_008010 & Fibroblast growth factor receptor 3 & Fgfr3 & 10.9 \\
\hline NM_008608 & Matrix metallopeptidase 14 & Mmp14 & 10.8 \\
\hline BB277517 & Histone deacetylase 7A & Hdac7a & 10.3 \\
\hline ВC016892 & Peroxisome proliferator receptor $\alpha$ & Ppara & 10.1 \\
\hline BG069466 & CREB binding protein & Crebbp & 9.8 \\
\hline NM_013565 & Integrin $\alpha 3$ & Itga3 & 9.6 \\
\hline ВB040443 & Snail homolog 2 (Drosophila) & Snai2 & 9.5 \\
\hline L07264 & Heparin-binding EGF-like growth factor & Hbegf & 9.4 \\
\hline AK004683 & Wingless-related MMTV integration 7A & Wnt7a & 8.8 \\
\hline BM239177 & Mitogen activated protein kinase 14 & Mapk14 & 8.7 \\
\hline ВC010202 & Kirsten rat sarcoma viral oncogene & Kras & 8.6 \\
\hline BB376407 & Jumonji, AT rich interactive domain 1A & Jarid1a & 8.2 \\
\hline BM119402 & Sloan-Kettering viral oncogene homolog & Ski & 7.7 \\
\hline NM_133249 & Peroxisome receptor coactivator $1 \beta$ & Ppargc $1 b$ & 7.1 \\
\hline ВC011118 & CCAAT/enhancer binding protein $\alpha$ & Cebpa & 6.8 \\
\hline NM_020265 & Dickkopf homolog 2 (Xenopus laevis) & Dkk2 & 6.8 \\
\hline NM_008416 & JunB oncogene & JunB & 6.5 \\
\hline AV264681 & Jagged 2 & Jag2 & 6.2 \\
\hline BG064099 & Braf transforming gene & Braf & 5.8 \\
\hline NM_007669 & Cyclin-dependent kinase inhibitor $1 \mathrm{~A}$ & Cdkn1a & -6.1 \\
\hline BF144658 & Transforming growth factor, $\beta 2$ & Tgfb2 & -6.7 \\
\hline AU043193 & Frizzled homolog 3 (Drosophila) & Fzd3 & -6.9 \\
\hline ВC008152 & Caspase 1 & Casp1 & -13.3 \\
\hline NM_009808 & Caspase 12 & Casp12 & -13.8 \\
\hline NM_011709 & Whey acidic protein & Wap & -22.1 \\
\hline ВC004601 & Casein $\kappa$ & Csn3 & -32.3 \\
\hline NM_010679 & Lactalbumin, $\alpha$ & Lalba & -212.2 \\
\hline NM_009972 & Casein $\beta$ & Csn & -310.8 \\
\hline NM_007784 & Casein $\alpha$ s1 & Csn1s1 & -365.2 \\
\hline
\end{tabular}


Table III. Differentially expressed genes between NBS1+/-;neu primary and metastatic tumors (222 genes).

\begin{tabular}{|c|c|c|c|}
\hline Accession & Gene name & Gene symbol & Fold change \\
\hline AV022238 & Chemokine (C-X-C motif) ligand 15 & Cxcl15 & 405.0 \\
\hline NM_010052 & $\Delta$-like 1 homolog (Drosophila) & Dlk1 & 308.8 \\
\hline ВС002073 & Chemokine (C-C motif) ligand 6 & $\mathrm{Ccl} 6$ & 113.2 \\
\hline NM_008471 & Keratin 19 & Krt19 & 111.5 \\
\hline AV304616 & Sonic hedgehog & Shh & 104.9 \\
\hline NM_010446 & Forkhead box A2 & Foxa2 & 81.7 \\
\hline NM_007554 & Bone morphogenetic protein 4 & Bmp4 & 69.4 \\
\hline NM_008010 & Fibroblast growth factor receptor 3 & Fgfr3 & 24.8 \\
\hline NM_007950 & Epiregulin & Ereg & 23.5 \\
\hline NM_008404 & Integrin $\beta 2$ & Itgb2 & 21.9 \\
\hline NM_011126 & Palate, lung and nasal carcinoma & Plunc & 20.1 \\
\hline AK007410 & Growth arrest and DNA-damage 45 & Gadd45g & 18.3 \\
\hline AV009267 & Forkhead box Q1 & Foxq1 & 18.1 \\
\hline ВC011118 & CCAAT/enhancer binding protein $\alpha$ & Cebpa & 15.4 \\
\hline AF416641 & Hypoxia inducible factor $3, \alpha$ subunit & Hif3a & 15.0 \\
\hline AA880220 & Jagged 1 & Jag1 & 13.9 \\
\hline AV322952 & Forkhead box P2 & Foxp2 & 12.8 \\
\hline NM_010207 & Fibroblast growth factor receptor 2 & Fgfr2 & 12.3 \\
\hline AV311104 & Cancer susceptibility candidate 4 & Casc4 & 11.3 \\
\hline BM231135 & Bone morphogenetic protein 1 & Bmp1 & 11.2 \\
\hline NM_013565 & Integrin $\alpha 3$ & Itga3 & 10.3 \\
\hline AV032115 & Bone morphogenetic protein 5 & Bmp5 & 8.6 \\
\hline AF128196 & Chemokine (C-C motif) ligand 9 & $\mathrm{Ccl} 9$ & 8.2 \\
\hline NM_008261 & Hepatic nuclear factor $4, \alpha$ & Hnf4a & 7.7 \\
\hline NM_011332 & Chemokine (C-C motif) ligand 17 & $\operatorname{Ccl} 17$ & 7.5 \\
\hline NM_008259 & Forkhead box A1 & Foxa1 & 7.3 \\
\hline BB373572 & Calcium dependent protein kinase II & Camk2d & 7.0 \\
\hline NM_020265 & Dickkopf homolog 2 (Xenopus laevis) & Dkk2 & 6.8 \\
\hline BB486740 & Hypoxia inducible factor $3, \alpha$ subunit & Hif3a & 6.7 \\
\hline BM293452 & Jumonji, AT rich interactive domain 2 & Jarid2 & 6.5 \\
\hline AV264681 & Jagged 2 & Jag2 & 6.2 \\
\hline M65143 & Lysyl oxidase & Lox & 6.0 \\
\hline NM_133654 & CD34 antigen & $\mathrm{Cd} 34$ & 6.0 \\
\hline ВС005453 & V-myc viral related oncogene & Mycn & 5.8 \\
\hline BE688115 & Fibroblast growth factor 1 & Fgf1 & 5.6 \\
\hline BB787243 & Insulin-like growth factor binding protein & Igfbp4 & 5.6 \\
\hline ВC023427 & Platelet derived growth factor, B & Pdgfb & 5.5 \\
\hline BB015508 & Jumonji domain containing $1 \mathrm{C}$ & Jmjd1c & 5.4 \\
\hline BB040443 & Snail homolog 2 (Drosophila) & Snai2 & 5.2 \\
\hline BQ175880 & Cyclin D2 & Ccnd2 & 5.0 \\
\hline BB543291 & Chemokine (C-C motif) receptor-like 1 & Ccrl1 & 5.0 \\
\hline NM_011121 & Polo-like kinase 1 (Drosophila) & Plk1 & -5.4 \\
\hline ВС027242 & Vav 3 oncogene & Vav3 & -5.8 \\
\hline X75483 & Cyclin A2 & Ccna2 & -5.8 \\
\hline ВС003261 & Aurora kinase B & Aurkb & -6.8 \\
\hline NM_009525 & Wingless-related integration site $5 \mathrm{~B}$ & Wnt5b & -6.9 \\
\hline BF144658 & Transforming growth factor, $\beta 2$ & Tgfb2 & -8.4 \\
\hline NM_019645 & Plakophilin 1 & Pkp1 & -8.4 \\
\hline AK013312 & Cyclin B2 & Ccnb2 & -8.4 \\
\hline AV367068 & Desert hedgehog & Dhh & -8.8 \\
\hline AU043193 & Frizzled homolog 3 (Drosophila) & Fzd3 & -10.5 \\
\hline NM_013505 & Desmocollin 2 & Dsc2 & -11.9 \\
\hline U03425 & Epidermal growth factor receptor & Egfr & -18.5 \\
\hline ВC014690 & Transforming growth factor, $\beta 3$ & Tgfb3 & -29.2 \\
\hline NM_007785 & Casein $\alpha$ s2-like A & $\mathrm{Csn} 1 \mathrm{~s} 2 \mathrm{a}$ & -127.4 \\
\hline NM_009972 & Casein $\beta$ & Csn & -160.8 \\
\hline NM_007786 & Casein $\kappa$ & $\mathrm{Csn} 3$ & -958.5 \\
\hline
\end{tabular}


A

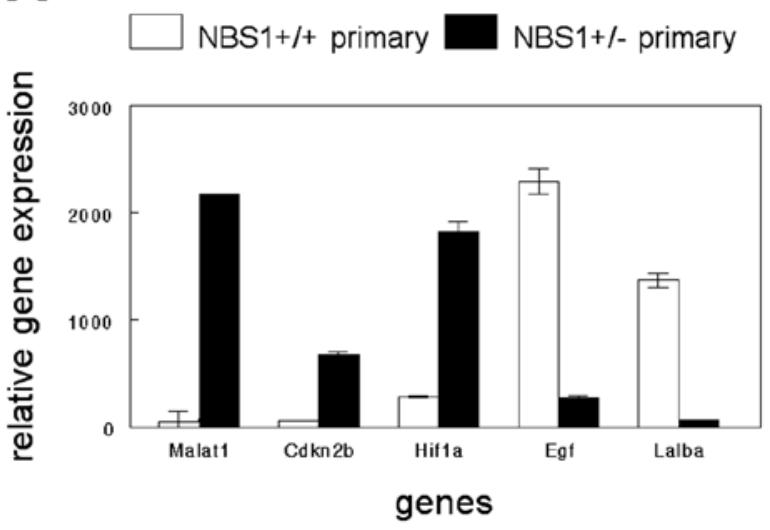

B
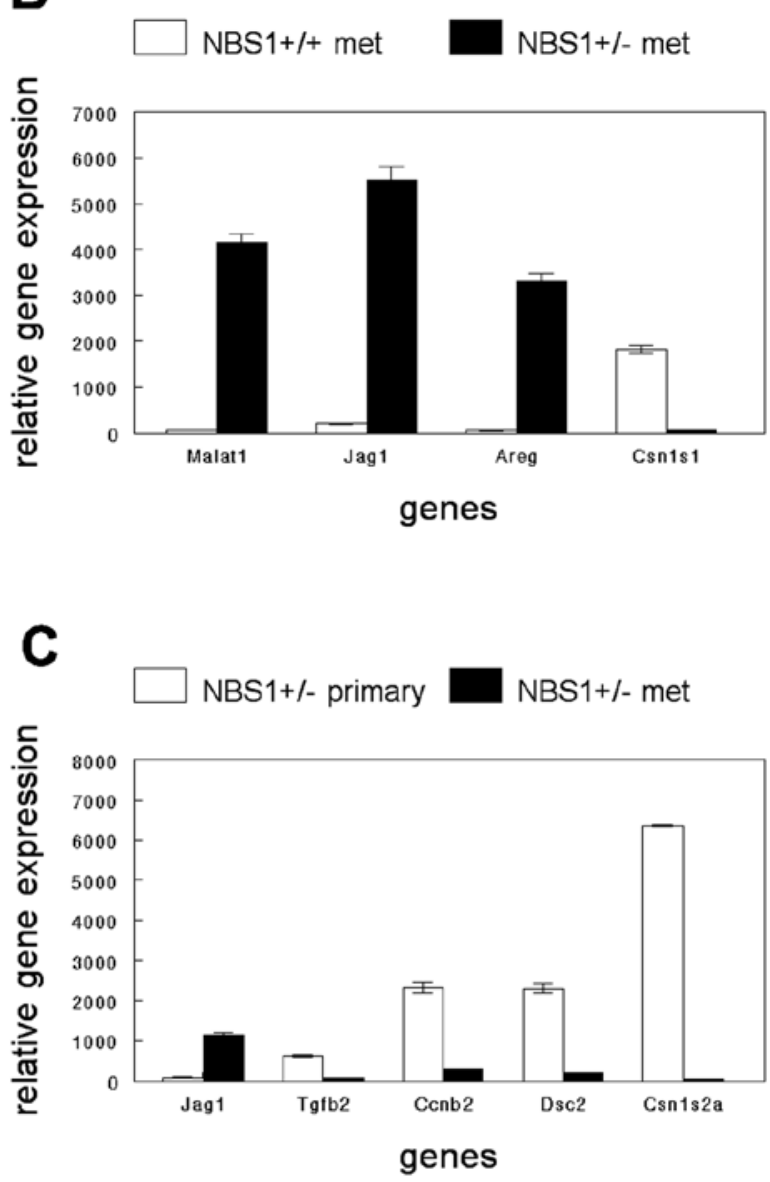

Figure 4. NBS1+/-;neu mammary tumors exhibit specific gene expression signatures. (A) Relative mRNA expression of genes in NBS1+/+;neu and NBS1+/-;neu primary mammary tumors was performed by qRT-PCR. (B) Relative mRNA expression of genes in NBS1+/+;neu and NBS1+/-;neu metastatic mammary tumors was performed by qRT-PCR. (C) Relative mRNA expression of genes in NBS1+/-;neu primary and metastatic mammary tumors was performed by qRT-PCR. Error bars indicate SEM.

bone morphogenetic protein signaling (Bmp4, 69.4-fold; Bmp1, 11.2-fold; Bmp5, 8.6-fold; Tgfb2, -8.4-fold), cell cycle (Ccnd2, 5-fold; Ccna2, -5.8-fold; Ccnb2, -8.4-fold), cell adhesion (Pkp1, -8.4-fold; Dsc2,-11.9-fold), forkhead signaling (Foxa2, 81.7-fold; Foxq1, 18.1-fold; Foxp2, 12.8-fold), epidermal growth factor signaling (Ereg, 23.5-fold; Egfr, -18.5-fold), and differentia- tion (Csn1s2a, -127.4-fold; Csn -160.8-fold; Csn3, -958.5-fold). Expression of representative genes involved in these pathways was validated by qRT-PCR in Fig. 4. These results demonstrated specific patterns of gene expression that correlated with primary and metastatic tumor phenotype.

\section{Discussion}

Mutations in the NBS1 gene have been associated with increased risk of breast cancer (14-16). Persistent radiation induced NBS1 foci has been associated with chromosomal instability and increased breast cancer risk (13). In mice, NBS1 null mutation is embryonic lethal but heterozygosity renders mice susceptible to tumor formation (5). However, mammary tumors are uncommon in mouse strains with reduced NBS1 function (6). To examine the role of NBS1 in mammary tumor formation, we examined NBS1 haploinsufficiency in the mammary tumor prone MMTV-neu strain. Reduced expression of NBS1 resulted in increased apoptosis in NBS1 heterozygous mice. This increased cell death correlated with markedly increased tumor latency in NBS1 heterozygous mice. These effects were likely due to decreased DNA repair following oncogene induced cellular proliferation. Defects in cellular proliferation were noted in the cells of NBS1 deficient mice in previous studies (6). Increased latency correlated with decreased growth factor expression and increased cyclin dependent kinase inhibitor expression. Loss of p53 has been shown to greatly increase tumorigenesis in NBS1 mutant mice, suggesting that p53 mediated DNA damage response may be responsible for apoptosis and increased tumor latency (15). A previous study demonstrated nuclear export of NBS1 following ionizing radiation as a mechanism of downregulating the DNA damage response (16). Previous studies have demonstrated increased chromosomal aberrations in NBS1+/- tumors (5). Loss of NBS1 has been shown to induce supernumerary centrosomes similar to those observed in BRCA1 deficient cells, leading to increased chromosomal instability (17). These studies demonstrate that impaired NBS1 function can result in cellular proliferation defects leading to increased tumor latency. It is interesting to speculate that tumorigenic clones that escape defective cell death pathways may be more aggressive and metastatic due to chromosomal aberrations induced by diminished NBS1 function. In support this hypothesis, increased numbers of differentially expressed genes and degregulated signaling pathways in NBS1+/-;neu tumors correlated with increased metastatic disease.

\section{Acknowledgements}

We thank Dr Yang Xu (University of California San Diego) for NBS1 mutant mice. This study was supported by Susan G. Komen for the Cure award BCTR0504295 and Department of Defense Breast Cancer Research Program award W81XWH10-1-0081 to D.L.C.

\section{References}

1. Barlev NA, Liu L, Chehab NH, Mansfield K, Harris KG, Halazonetis TD and Berger SL: Acetylation of p53 activates transcription through recruitment of coactivators/histone acetyltransferases. Mol Cell 8: 1243-1254, 2001. 
2. Bartkova J, Tommiska J, Oplustilova L, Aaltonen K, Tamminen A, Heikkinen T, Mistrik M, Aittomaki K, Blomqvist C, Heikkila P, Lukas J, Nevanlinna H and Bartek J: Aberrations of the MRE11RAD50-NBS1 DNA damage sensor complex in human breast cancer: MRE11 as a candidate familial cancer predisposing gene. Mol Oncol 2: 296-316, 2008.

3. Bogdanova N, Feschchenko S, Schurmann P, Waltes R, Wieland B, Hillemanns P, Rogov YI, Dammann O, Bremer M, Karstens JH, Sohn C, Varon R and Dork T: Nijmegen breakage syndrome mutations and risk of breast cancer. Int J Cancer 122: 802-806, 2008.

4. Cai BH, Chen JY, Lu MH, Chang LT, Lin HC, Chang YM and Chao CF: Functional four base A/T gap core sequence CATTAG of p53 response elements specifically bound tetrameric p53 differently than two base A/T gap core sequence CATG bound both dimeric and tetrameric p53. Nuc Acids Res 37: 1984-1990, 2009.

5. Chiang YC, Teng SC, Su YN, Hsieh FJ and Wu KJ: c-myc directly regulates the transcription of the NBS1 gene involved in DNA double strand break repair. J Biol Chem 278: 19286-19291, 2003.

6. Crowe DL and Lee MK: New role for nuclear hormone receptors and coactivators in regulation of BRCA1 mediated DNA repair in breast cancer cell lines. Breast Cancer Res 8: 1-12, 2006.

7. Dumon-Jones V, Frappart PO, Tong WM, Sajithlal G, Hulla W, Schmid G, Herceg Z, Digweed M and Wang ZQ: Nbn heterozygosity renders mice susceptible to tumor formation and ionizing radiation induced tumorigenesis. Cancer Res 63: 7263-7269, 2003.

8. Eggleston $\mathrm{P}$ and Zhao Y: A sensitive and rapid assay for homologous recombination in mosquito cells: impact of vector topology and implications for gene targeting. BMC Genet 2: 21-29, 2001.
9. Frappart PO, Tong WM, Demuth I, Radovanovic I, Herceg Z, Aguzzi A, Digweed M and Wang ZQ: An essential function for NBS1 in the prevention of ataxia and cerebellar defects. Nature Med 11: 538-544, 2005.

10. Goodman RH and Smolik S: CBP/p300 in cell growth, transformation, and development. Genes Dev 14: 1553-1577, 2000.

11. Hakem R: DNA damage repair; the good, the bad, and the ugly. EMBO J 27: 589-605, 2008.

12. Hsu HM, Wang HC, Chen ST, Hsu GC, Shen CY and Yu JC: Breast cancer risk is associated with the genes encoding the DNA double strand break repair Mre11/Rad50/Nbs1 complex. Cancer Epidemiol Biomarkers Prev 16: 2024-2032, 2007.

13. Kang J, Bronson RT and Xu Y: Targeted disruption of NBS reveals its roles in mouse development and DNA repair. EMBO J 21: 1447-1455, 2002

14. Nowak J, Mosor M, Ziolkowska I, Wierzbicka M, PernakSchwarz M, Przyborska M, et al: Heterozygous carriers of the I171V mutation of the NBS1 gene have a significantly increased risk of solid malignant tumors. Eur J Cancer 44: 627-630, 2008.

15. Kang J, Ferguson D, Song H, Bassing C, Eckersdorff M, Alt FW and $\mathrm{Xu}$ Y: Functional interaction of $\mathrm{H} 2 \mathrm{AX}, \mathrm{NBS1}$, and p53 in ATM dependent DNA damage responses and tumor suppression. Mol Cell Biol 25: 661-670, 2005.

16. Vissinga CS, Yeo TC, Warren S, Brawley JV, Phillips J, Cerosaletti $K$ and Concannon P: Nuclear export of NBN is required for normal cellular responses to radiation. Mol Cell Biol 29: 1000-1006, 2009.

17. Shimada M, Sagae R, Kobayashi J, Habu T and Komatsu K: Inactivation of the Nijmegen breakage syndrome gene leads to excess centrosome duplication via the ATR/BRCA1 pathway. Cancer Res 69: 1768-1775, 2009. 